\title{
Postoperative liver metastasis of primary inflammatory pseudotumorous follicular dendritic cell sarcoma of the spleen: a case report
}

\author{
Run-Hua Pang^, Ya-Qing Zhu, Jian Wu, Jun-Hai Huang, Bin Chen \\ Department of Hepatobiliary Surgery, the First Affiliated Hospital of Guangzhou University of Traditional Chinese Medicine, Guangzhou, China \\ Correspondence to: Ya-Qing Zhu. Department of Hepatobiliary Surgery, the First Affiliated Hospital of Guangzhou University of Traditional Chinese \\ Medicine, 16 Jichang Road, Baiyun District, Guangzhou 510405, China. Email: doctorzhuyq@163.com.
}

\begin{abstract}
Follicular dendritic cell sarcoma (FDCS) is a rare and low incidence tumor. So far, there is no standard treatment for the disease. Surgery is the main treatment for FDCS. Here, we report the case of a 51-year-old woman who was admitted with the chief complaint of "spleen-occupying lesion detected via physical examination more than 1 month ago". Spiral plain scan and enhanced computed tomography (CT) of the upper abdomen showed a vascular-derived tumor, with a high possibility of hemangioma or hemangiolymphangioma. Later, on 25 December 2019, the patient underwent laparoscopic splenectomy, and the pathological diagnosis of primary splenic FDCS was made. The patient did not receive adjuvant chemotherapy or radiotherapy. At a regular follow-up inspection 11 months later, on 18 December 2020, an abdominal scan + enhanced CT revealed multiple new abnormal intrahepatic nodules, which combined with her history, indicated the possibility of metastases. Subsequently, she was readmitted to hospital and surgical treatment was decided upon after multi-disciplinary consultation and discussion. Laparoscopic S1/S3/S4/ S5 hepatectomy plus cholecystectomy was performed on 31 December 2020. The postoperative pathology findings revealed (liver tumor, S3, S4, S5) metastatic inflammatory pseudotumor follicular dendritic sarcoma, tumor diameter $0.6-1.2 \mathrm{~cm}$. Due to the lack of clinical reports on postoperative organ metastasis of this disease, less experience, and controversy in drug selection of radiotherapy and chemotherapy, the patient refused to receive radiotherapy and chemotherapy. She now undertakes regular outpatient reexamination, and has been followed-up until now, during which time she has not progressed, and the efficacy is considered satisfactory.
\end{abstract}

Keywords! Follicular dendritic cell sarcoma (FDCS); liver metastases; surgical treatment; case report

Submitted Jul 09, 2021. Accepted for publication Aug 18, 2021.

doi: 10.21037/apm-21-2155

View this article at: https://dx.doi.org/10.21037/apm-21-2155

\section{Introduction}

Follicular dendritic cell sarcoma (FDCS) is a very rare malignant tumor for which the pathogenesis remains unclear. It is mainly derived from dendritic cells and has clinical histological and pathological characteristics similar to those of an inflammatory pseudotumor (1), and plays an important role in antigen presentation to $\mathrm{B}$ cells in lymphatic organs (2). The incidence of FDCS is relatively low. There have been few single cases and case series of FDCS in literature from China and worldwide. To date, no cases of liver metastasis after splenic FDCS have been reported so far. For the treatment of primary FDCS, surgery is the preferred treatment; however, FDCS recurrence and distant metastasis is the main cause of treatment failure. For the treatment of metastatic FDCS, research $(3,4)$ has

$\wedge$ ORCID: 0000-0001-8725-6204. 
indicated that systemic chemotherapy has certain benefits, but it has remained controversial, and there is no uniform standard. In this paper, we have described the circumstance of a spleen FDCS patient after resection, postoperative follow-up of 11 months, occurrence of liver metastatic FDCS, surgical resection, and with the ongoing followup, no further recurrence to date. We present the following article in accordance with the CARE reporting checklist (available at https://dx.doi.org/10.21037/apm-21-2155).

\section{Case presentation}

All procedures performed in studies involving human participants were in accordance with the ethical standards of the institutional and/or national research committee(s) and with the Helsinki Declaration (as revised in 2013). Written informed consent was obtained from the patient for publication of this case report and accompanying images. A copy of the written consent is available for review by the editorial office of this journal.

\section{Chief complaints}

A 51-year-old female patient with an inflammatory pseudotumorous FDCS of the spleen was diagnosed with a liver space occupying lesion upon physical examination 11 months after surgery.

\section{History of present illness}

A 51-year-old Chinese woman presented at the hospital on 19 December 2019 for medical examination after a spleenoccupying lesion was detected 1 month before admission. According to laboratory examination, serum tumor markers (AFP, CEA, CA125, and CA19-9) were negative. Her imaging examination involved an abdominal spiral scan + enhanced computed tomography (CT), which revealed increased spleen volume, a lamellar irregular low-density shadow in essence, with unclear boundary, calcifications, within a range of about $50 \mathrm{~mm} \times 31 \mathrm{~mm}$, enhanced scan a gradual strengthening. Magnetic resonance imaging (MRI) plain scan + enhancement showed splenomegaly, oval mixed long and short T1 and long T2 signals in the central region, and uneven high signal on diffusion weighted imaging (DWI). Enhanced scanning showed edge enhancement in arterial phase, enhanced filling to the center in portal phase and delayed phase, with a size of about $37 \times 46 \times 29 \mathrm{~mm}^{3}$. A diagnosis was made of vascular tumor, with likelihood of hemangioma or hemangiolymphangioma. After the preoperative examination was completed, laparoscopic splenectomy was performed on 25 December 2019. Postoperative pathology revealed an inflammatory pseudotumorous FDCS of the spleen. The patient did not receive adjuvant chemotherapy or radiotherapy after surgery, and regular follow-up in the outpatient department for more than 11 months showed no obvious abnormalities until 28 December 2021. Total abdominal plain scan + enhanced CT showed: postoperative changes of the spleen, small round and slightly low-density shadows were observed at the subcapsular S3, S4, and S5 areas of the liver, with the largest being $10 \mathrm{~mm} \times 11 \mathrm{~mm}$ in size. Combined with her medical history, the possibility of metastatic tumor was considered. The patient was admitted for further evaluation and treatment.

\section{Physical examination upon admission}

Vital signs such as respiration, pulse, temperature, and blood pressure were all within the normal range. There were no other positive signs.

\section{Laboratory examinations}

The laboratory indicators at both points of hospitalization2 hospitalized patients showed normal white blood cell count, hemoglobin and platelet count, normal liver function and biochemical indexes, and negative serum tumor markers (AFP, CEA, CA125, and CA19-9).

\section{Imaging examinations}

On the first admission of the patient (24 December 2019), the spiral plain scan of the upper abdomen and enhanced CT showed that the spleen had increased in volume, irregular low-density patchy shadowing was observed in the tissue, the boundary was not clear, and calcification was observed, with a range of about $50 \mathrm{~mm} \times 31 \mathrm{~mm}$ (Figure 1). Plain MRI + enhanced MRI showed splenomegaly with a size of $37 \times 46 \times 29 \mathrm{~mm}^{3}$ (Figure 2).

On the second admission of the patient (28 December 2020), abdominal plain scan + enhanced CT showed: postoperative changes of spleen, multiple hepatic cysts, small gallbladder stones, and multiple abnormal enhancement shadows in the liver. Small round and slightly low-density shadows were observed under the lobes of S3, S4, and S5 segments of the liver, and the larger ones were 

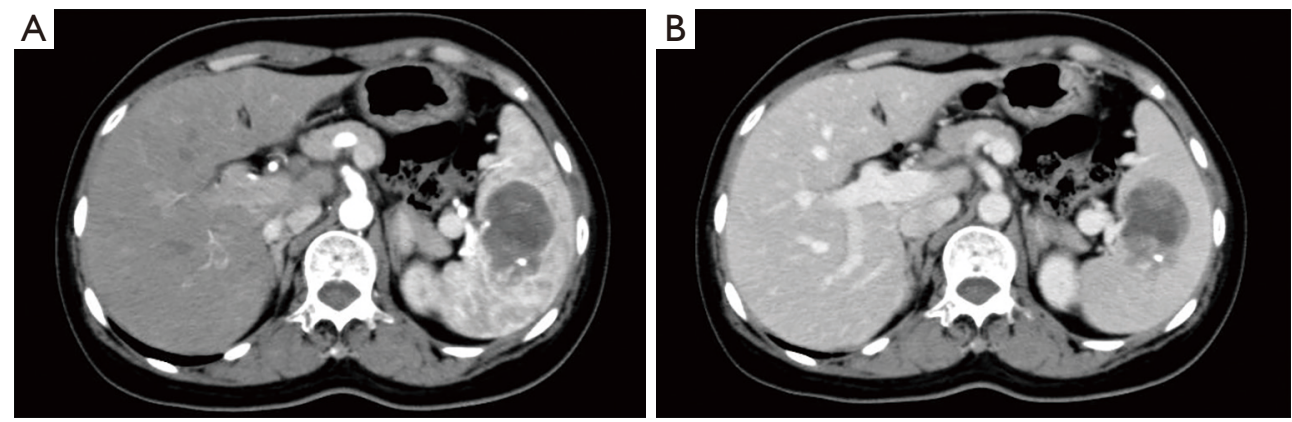

Figure 1 Upper abdominal plain scan + enhanced CT. (A) Intermediate lesions (arrow) of the spleen were observed in the enhanced phase, with unclear boundaries and uneven enhancement around, with a range of about $50 \mathrm{~mm} \times 31 \mathrm{~mm}$. Enhanced scan showed progressive enhancement. (B) In the venous phase, the intermediate lesion (arrow) of the spleen was strengthened at the center, which was consistent with the imaging findings of "fast entry and slow exit".
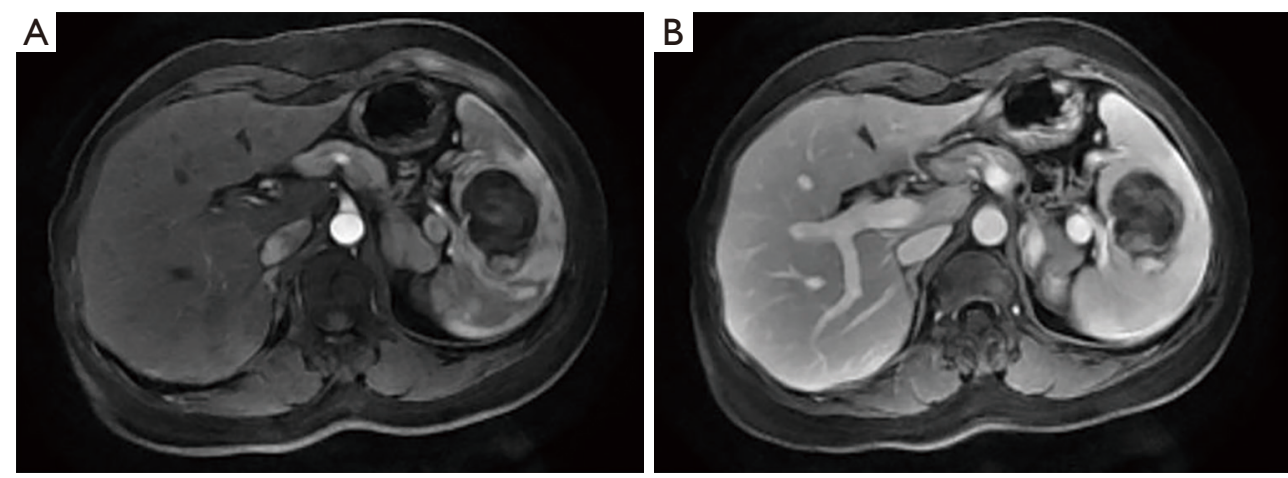

Figure 2 Plain and enhanced MRI of liver, gallbladder, spleen, and pancreas. (A) DWI shows uneven hyperintensity, oval lesion in the center of the spleen with a size of about $37 \times 46 \times 29 \mathrm{~mm}^{3}$. Enhanced scanning shows marginal enhancement in the arterial phase. (B) Portal phase and delayed phase enhanced filling to the center, no enhancement was observed in the central cystic component of the lesion. Consistent with the imaging findings of "fast entry and slow exit". MRI, magnetic resonance imaging; DWI, diffusion weighted imaging.

about $10 \mathrm{~mm} \times 11 \mathrm{~mm}$. The enhancement presented slight enhancement (Figure 3), and the possibility of metastasis was considered based on the medical history.

\section{Final diagnosis}

The postoperative pathology of the first operation (Figure 4) was as follows: (I) inflammatory pseudotumor FDCS of the spleen; (II) splenic hilum tissues and lymph nodes, with the following immunohistochemical (IHC) expression: CD21 (+), CD23 (+), SMA (-), S-100 (-), ALK (-), CD34 (-), Ki67 (+ 10\%), and CD8 (+) background lymphocytes.

Postoperative pathology of the second operation (Figure 5) was as follows: (I) (liver tumor, S3, S4, and S5 segments) metastatic inflammatory hypotumor-like FDCS, tumor diameter 0.6-1.2 cm; (II) chronic calculous cholecystitis; (III) (S1 caudate lobe) mild chronic hepatitis, no tumor observed. The IHC was as follows: CD21 (+++), CD23 (+++), CD34 (-), S-100 (-), ALK (-), SMA (+), Ki-67 (+ 30\%).

\section{Treatment}

Laparoscopic splenectomy was performed on the first admission, and laparoscopic S1/S3/S4/S5 hepatectomy was performed on the second admission.

\section{Outcome and follow-up}

After the first operation, the patient did not receive any radiotherapy or chemotherapy. After regular outpatient 

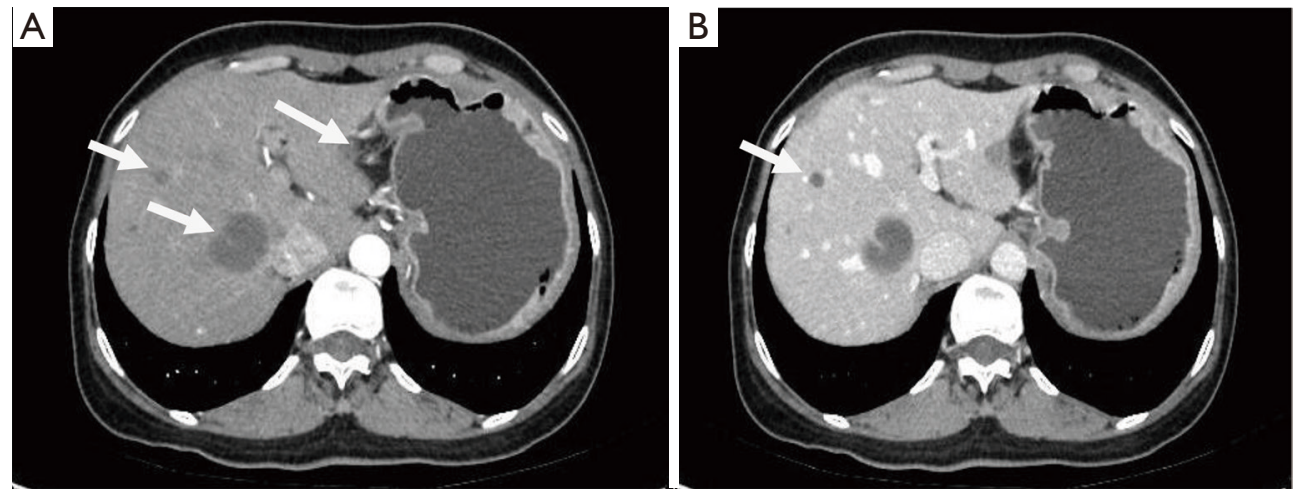

Figure 3 Full abdominal plain scan + enhanced CT. (A) Round low density shadow of caudate lobe, clear boundary, no enhancement observed. Small round shadow (arrow) with slightly lower density was seen under the liver S3 and S5 capsule, and the larger one was about $10 \mathrm{~mm} \times 11 \mathrm{~mm}$, with slight enhancement. (B) S4 lesion (arrow) with mild enhancement.
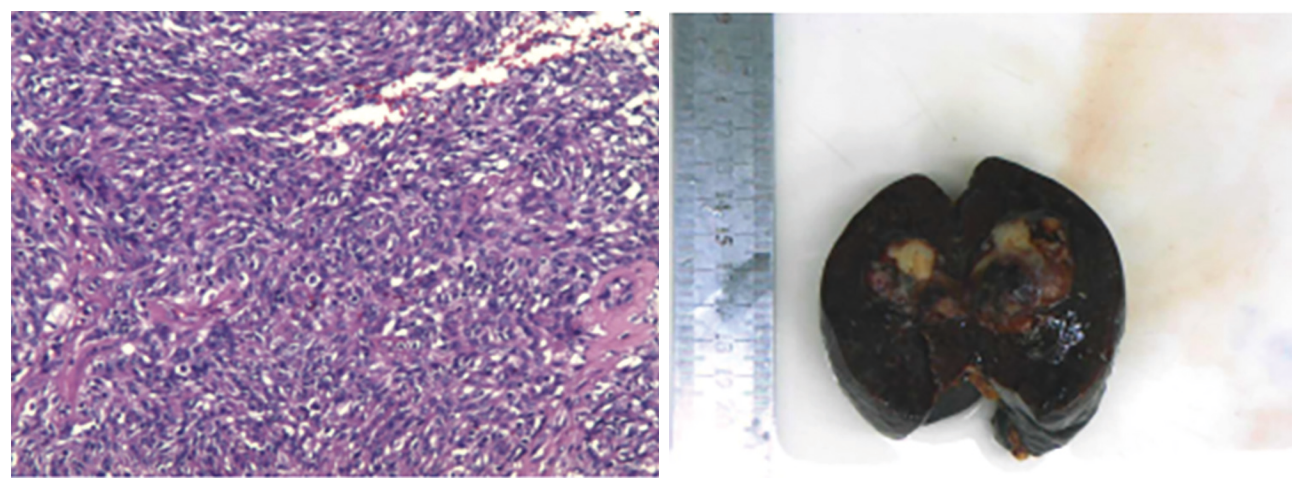

Figure 4 Primary splenic tumor, HE staining (enlarged view, $\times 200$ ). Tumor tissue boundary is unclear, tumor cells are arranged in spindle, braided, and swirling shape, cell atypia is light, nuclear traces can be seen, nuclear mitosis can be seen, focal bleeding and collagen fiber proliferation can be seen, a large number of plasma cells, lymphocytes infiltration. HE, hematoxylin and eosin.
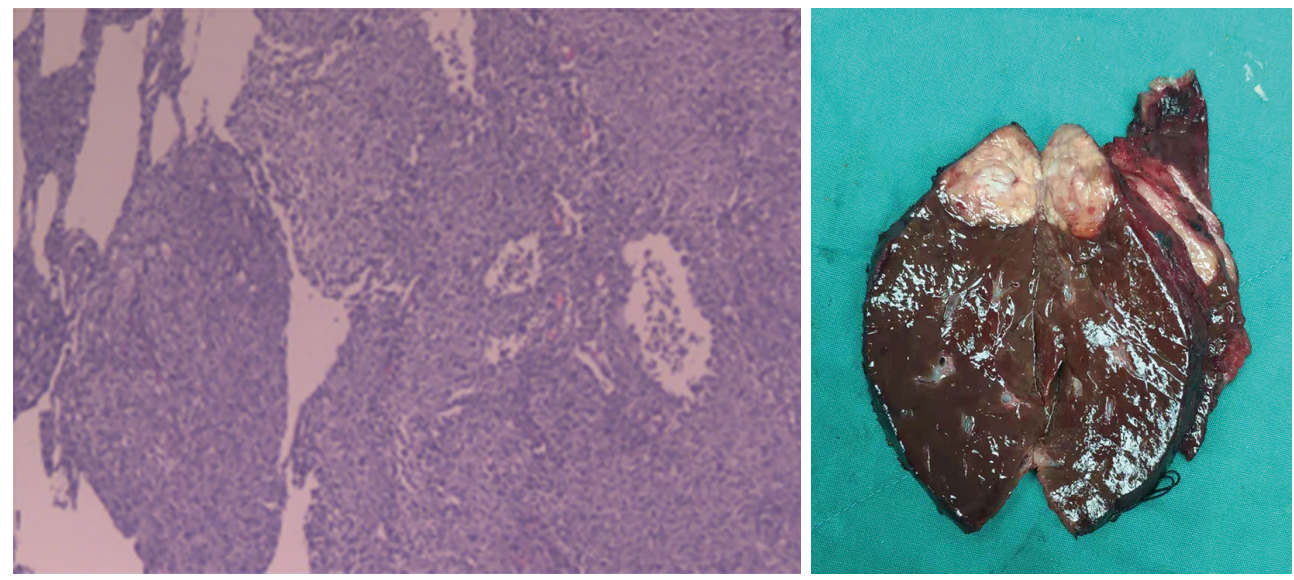

Figure 5 Metastatic tumor of spleen, HE staining (enlarged view, ×200). The proliferation of tumor cells is nodular, surrounded by lymphocyte infiltration. The tumor cells are not clearly demarcated and local were fusiform. The nuclei are large and pale, and scattered lymphocyte infiltration is seen inside. HE, hematoxylin and eosin. 
follow-up for 11 months, the patient was discharged from hospital for liver metastasis. Subsequently, the patient received radical hepatectomy on the second admission.

\section{Discussion}

According to the Fourth Revised Edition of the World Health Organization Classification of Hematopoietic and Lymphoid Tissue Tumors published in 2017, FDCS is defined as "a proliferation of spindle-shaped to oval cells with FDC morphology and immunophenotypic characteristics" (5). In 1997, Chan et al. (6) showed that FDCS was a multifaceted tumor with rare etiological association. The combination of light microscopy, ultrastructure, and IHC characteristics are considered to have a common source with follicular dendritic cells (FDC) in lymphoid follicles. In 1986, Monda et al. (7) first reported 4 cases of special lymph nodes, which were differentiated by dendritic cells. In 2000, they and later Toyoda et al. (8) reported a special tumor, which showed a rare non lymphoid (or non-lymphoma) primary lymph node nausea tumor, which was reported to be from a 71-year-old female patient. In 2002, van Nierop et al. (9) published a paper in which he described in detail the origin, characteristics and pathological process of $\mathrm{FDC}_{\mathrm{S}}$, in which they showed that FDCs are non-lymphoid antigen presenting cells and play an important role in antigen presentation related to memory B cells. FDCs can form immune complexes, which can not only preserve and protect the antigens of B cell function, but also promote the growth and differentiation of B cells (10). A large study published by Saygin et al. (11) in 2013 showed that lymph nodes (neck, axilla, and mediastinum) were involved in slightly more than $30 \%$ of cases, extrinsic organs were involved in slightly less than $60 \%$ of cases, and about $10 \%$ of patients had lymph nodes and extrinsic lesions. Extranodal FDCs usually involve the gastrointestinal tract, tonsil, nasopharynx, local tissue, lung, omental tissue, etc. (12). It can occur in the liver and spleen, often complicated by Epstein-Barr virus infection in most patients with single lesions. For patients with clinically visible symptoms such as abdominal distension, abdominal pain, and fever, diagnosis is made via physical examination and imaging findings, due to the lack of obvious specificity in clinical manifestation $(13,14)$. The age range for FDCS is wide, but it mainly occurs in adults. The median age at diagnosis of FDCS is about 45-50 years old, and the distribution of men and women is basically the same $(8,11)$. Li et al. (15) published an article on 5 cases of FDCs, which came from the abdomen of 5 patients. Interestingly, the CT findings of these patients' lesions. They believe that caution should be taken in the diagnosis of FDCs when they are large, well or poorly demarcated, have complex internal structures, have significant internal necrosis and/or focal calcification, and have heterogeneous enhancement of solid components. Histologically, FDCs consist of bundles, spirals (similar to meningiomas), and fusiform, ovate, and elongated cells with eosinophilic cytoplasm, ovoid nuclei, vesicular or finely dispersed chromatin, small and well defined nucleoli, and ill-defined cell boundaries. Cellular atypia is uncommon, but can be seen in some cases. Mitotic activity ranges from 0 to 10 mitoses per 10 hyperploidy fields, but some cases may exhibit higher mitotic activity with significant nuclear atypical and focal necrosis in this case, the pathological findings of the patient showed that the tumor cells were fusiform, braided, and swirling, with mild cell atypia and a large number of plasma cells and lymphocytes infiltration, which was consistent with the literature. In more than $90 \%$ of cases, there are usually small lymphocytes and plasma cells clustered around the blood vessels. Pseudoinclusions generally exist in tumor cells, with rich types. There are various giant cells such as double nucleus and multicellular nucleus. These morphological characteristics have been recognized by a large number of studies and WHO (16-21). In terms of IHC, FDCs express CD21, CD23, and CD35 as well as cluster proteins (other dendritic cell tumors are usually negative). Other common expression markers include vimentin, fascial protein, podoplanin, desmoplakin, EGFR, and CXL13. Additionally, S100 protein, EMA and CD68 were mutated and expressed in FDCS. Cytokeratin, CD1A, CD3, CD79A, CD34, $\mathrm{MPO}$, and so on of FDCs are usually negative. The average Ki67 proliferation index has been shown to be about $13 \%$. The molecules CD21, CD23, and CD35 are considered markers of FDC differentiation and have high sensitivity and specificity for the diagnosis of FDCS. This paper reported the patients' IHC, and the first assessment showed: CD21 (+), CD23 (+), SMA (-), S-100 (-), ALK (-), CD34 $(-)$ Ki-67 (+ 10\%); the second IHC results were as follows: CD2 $1(+++)$, CD23 (+++), CD34 (-), S-100 (-), ALK $(-)$, SMA (+), and Ki-67 (+ 30\%). Both CD21 and CD23 were positive, and $\mathrm{CD} 34$ was negative. The average Ki67 proliferation index was $20 \%$, which was basically consistent with the reported literature. The above IHC description of FDCs have been recognized by a large number of studies and WHO (21-23). Transcriptional profiles have suggesting monitoring the expression of programmed death ligands-1 
and 2 (PD-L1 and PD-L2), as they may contribute to the development of these tumors. The genes encoding follicular dendritic cell secretory proteins (FDCSP) and serglycin (SRGN) are expressed in FDCs with high specificity, and IHC markers of these proteins are available, which play an important role in diagnosis and have more sensitivity and specificity than most markers. Therefore, they can improve the diagnostic rate of FDCs $(24,25)$. The general prognosis of FDCS is considered to be moderate, and that of localized tumors is good. Although complete surgical resection is the most widely used form of treatment, due to the rarity of these tumors, at present, there is no internationally recognized treatment guideline. The mechanism of the therapeutic effect of radiotherapy or chemotherapy on FDCs is unclear. Surgical resection has been the primary method for more than $90 \%$ of localized tumors $(22,24)$. The largest study to date was conducted by Saygin et al., which reported recurrence rates of $22 \%$ and metastases rates of $27 \%$. Pulmonary metastasis from cervical FDCS has been reported in the literature $(25,26)$. The case reported in this paper is the first to document the diagnosis of splenic inflammatory pseudotumor, FDCS treated by surgical resection, and subsequent postoperative liver metastasis yet with the splenic inflammatory pseudotumor FDCS of liver metastasis occurred after reports, this paper first inflammatory pseudotumor-like the FDCS postoperative liver metastases, and were treated with surgery, regular follow-up after resection of the metastatic lesions, did not see a relapse, and a satisfactory curative effect was seen.

\section{Conclusions}

There is currently no standard handling of FDCS. Based on the literature and the results obtained in our case, surgical resection may present a reliable option for the treatment of metastatic hepatic FDCS.

\section{Acknowledgments}

Funding: None.

\section{Footnote}

Reporting Checklist: The authors have completed the CARE reporting checklist. Available at https://dx.doi. org/10.21037/apm-21-2155

Conflicts of Interest: All authors have completed the ICMJE uniform disclosure form (available at https://dx.doi. org/10.21037/apm-21-2155). The authors have no conflicts of interest to declare.

Ethical Statement: The authors are accountable for all aspects of the work in ensuring that questions related to the accuracy or integrity of any part of the work are appropriately investigated and resolved. All procedures performed in studies involving human participants were in accordance with the ethical standards of the institutional and/or national research committee(s) and with the Helsinki Declaration (as revised in 2013). Written informed consent was obtained from the patient for publication of this case report and accompanying images. A copy of the written consent is available for review by the editorial office of this journal.

Open Access Statement: This is an Open Access article distributed in accordance with the Creative Commons Attribution-NonCommercial-NoDerivs 4.0 International License (CC BY-NC-ND 4.0), which permits the noncommercial replication and distribution of the article with the strict proviso that no changes or edits are made and the original work is properly cited (including links to both the formal publication through the relevant DOI and the license). See: https://creativecommons.org/licenses/by-nc-nd/4.0/.

\section{References}

1. Martins PN, Reddy S, Martins AB, et al. Follicular dendritic cell sarcoma of the liver: unusual presentation of a rare tumor and literature review. Hepatobiliary Pancreat Dis Int 2011;10:443-5.

2. Ohtake H, Yamakawa M. Interdigitating dendritic cell sarcoma and follicular dendritic cell sarcoma: histopathological findings for differential diagnosis. J Clin Exp Hematop 2013;53:179-84.

3. Shinagare AB, Ramaiya NH, Jagannathan JP, et al. Primary follicular dendritic cell sarcoma of liver treated with cyclophosphamide, doxorubicin, vincristine, and prednisone regimen and surgery. J Clin Oncol 2011;29:e849-51.

4. Jain P, Milgrom SA, Patel KP, et al. Characteristics, management, and outcomes of patients with follicular dendritic cell sarcoma. Br J Haematol 2017;178:403-12.

5. Chan JKC, Pileri SA, Fletcher CDM, et al. WHO classification of tumours of haematopoitic and lymphoid tissues. revised 4th ed. Lyon: International Agency for 
Research on Cancer; 2017:476-8.

6. Chan JK. Proliferative lesions of follicular dendritic (cells: an overview, including a detailed account of follicular dendritic cell sarcoma, a neoplasm with many faces and uncommon etiologic associations. Adv Anat Pathol 1997;4:387-411.

7. Monda L, Warnke R, Rosai J. A primary lymph node malignancy with features suggestive of dendritic reticulum cell differentiation. A report of 4 cases. Am J Pathol 1986;122:562-72.

8. Toyoda K, Taniguchi J, Kikawa K, et al. Follicular dendritic cell sarcoma: ultrastructural and immunohistochemical studies. Intern Med 2000;39:950-5.

9. van Nierop K, de Groot C. Human follicular dendritic cells: function, origin and development. Semin Immunol 2002;14:251-7.

10. Choi BS, Baek JH, Shin YM, et al. Follicular dendritic cell sarcoma: a case report and review of the literature. Cancer Res Treat 2010;42:121-4.

11. Saygin C, Uzunaslan D, Ozguroglu M, et al. Dendritic cell sarcoma: a pooled analysis including 462 cases with presentation of our case series. Crit Rev Oncol Hematol 2013;88:253-71.

12. Yuan T, Yang Q, Zhang H, et al. A 46-year-old Chinese woman presenting with retroperitoneal follicular dendritic cell sarcoma: a case report. J Med Case Rep 2014;8:113.

13. Van Baeten C, Van Dorpe J. Splenic Epstein-Barr VirusAssociated Inflammatory Pseudotumor. Arch Pathol Lab Med 2017;141:722-7.

14. Li XQ, Cheuk W, Lam PW, et al. Inflammatory pseudotumor-like follicular dendritic cell tumor of liver and spleen: granulomatous and eosinophil-rich variants mimicking inflammatory or infective lesions. Am J Surg Pathol 2014;38:646-53.

15. Li J, Geng ZJ, Xie CM, et al. Computer Tomography Imaging Findings of Abdominal Follicular Dendritic Cell Sarcoma: A Report of 5 Cases. Medicine (Baltimore) 2016;95:e2404.

16. Biddle DA, Ro JY, Yoon GS, et al. Extranodal follicular dendritic cell sarcoma of the head and neck region: three

Cite this article as: Pang $\mathrm{RH}, \mathrm{Zhu} \mathrm{YQ}, \mathrm{Wu} \mathrm{J}$, Huang JH, Chen B. Postoperative liver metastasis of primary inflammatory pseudotumorous follicular dendritic cell sarcoma of the spleen: a case report. Ann Palliat Med 2021;10(8):92979303. doi: 10.21037/apm-21-2155 new cases, with a review of the literature. Mod Pathol 2002;15:50-8.

17. Han JH, Kim SH, Noh SH, et al. Follicular dendritic cell sarcoma presenting as a submucosal tumor of the stomach. Arch Pathol Lab Med 2000;124:1693-6.

18. Shen DP, Ni XZ, Yin XL, et al. Clinical and pathological features of follicular dendritic cell sarcoma of appendix: a case report. Chin Med J (Engl) 2009;122:1595-7.

19. Choi JW, Lee JH, Kim A, et al. Follicular dendritic cell sarcoma arising in the dura mater of the spine. Arch Pathol Lab Med 2006;130:1718-21.

20. Chan JK, Fletcher CD, Nayler SJ, et al. Follicular dendritic cell sarcoma. Clinicopathologic analysis of 17 cases suggesting a malignant potential higher than currently recognized. Cancer 1997;79:294-313.

21. Schraven SP, Plontke SK, Syha R, et al. Dendritic cell tumor in a salivary gland lymph node: a rare differential diagnosis of salivary gland neoplasms. Diagn Pathol 2011;6:94.

22. Dalia S, Shao H, Sagatys E, et al. Dendritic cell and histiocytic neoplasms: biology, diagnosis, and treatment. Cancer Control 2014;21:290-300.

23. Perkins SM, Shinohara ET. Interdigitating and follicular dendritic cell sarcomas: a SEER analysis. Am J Clin Oncol 2013;36:395-8.

24. Lorenzi L, Döring C, Rausch T, et al. Identification of novel follicular dendritic cell sarcoma markers, FDCSP and SRGN, by whole transcriptome sequencing. Oncotarget 2017;8:16463-72.

25. Griffin GK, Sholl LM, Lindeman NI, et al. Targeted genomic sequencing of follicular dendritic cell sarcoma reveals recurrent alterations in $\mathrm{NF}$ - B regulatory genes. Mod Pathol 2016;29:67-74.

26. Chan AC, Chan KW, Chan JK, et al. Development of follicular dendritic cell sarcoma in hyaline-vascular Castleman's disease of the nasopharynx: tracing its evolution by sequential biopsies. Histopathology 2001;38:510-8.

(English Language Editor: J. Jones) 